\title{
Variations saisonnières des formes de phosphores dans une station de traitement des eaux usées par lagunage, sous climat aride de Marrakech (Maroc) \\ Seasonal variations in the forms of phosphorus in a wastewater stabilization pond under arid climatic conditions at Marrakech (Morocco)
}

\author{
N. Ouazzani, L. Bouarab, B. Picot, H. B. Lazrek, B. Oudra et J. Bontoux
}

Volume 10, numéro 4, 1997

URI : https://id.erudit.org/iderudit/705292ar

DOI : https://doi.org/10.7202/705292ar

Aller au sommaire du numéro

Éditeur(s)

Université du Québec - INRS-Eau, Terre et Environnement (INRS-ETE)

ISSN

0992-7158 (imprimé)

1718-8598 (numérique)

Découvrir la revue

Citer cet article

Ouazzani, N., Bouarab, L., Picot, B., Lazrek, H. B., Oudra, B. \& Bontoux, J. (1997).

Variations saisonnières des formes de phosphores dans une station de

traitement des eaux usées par lagunage, sous climat aride de Marrakech

(Maroc). Revue des sciences de l'eau / Journal of Water Science, 10(4), 527-544.

https://doi.org/10.7202/705292ar

\section{Résumé de l'article}

L'objectif de ce travail est d'étudier dans un premier temps la dynamique du phosphore dans un système de traitement $d$ 'eaux usées par lagunage facultatif, en relation avec les compartiments biologiques: phytoplancton, zooplancton et bactéries, dans un deuxième temps, d'évaluer, sous les conditions du climat aride, les performances épuratoires de ce système en ce qui concerne les formes du phosphore. Un intérêt particulier est porté au phénomène du relargage du phosphore par les sédiments.

Les résultats montrent qu'il y a une évolution saisonnière et cyclique du phosphore dans les bassins de lagunage, en étroite corrélation avec le phytoplancton et le zooplancton. Le phytoplancton représente la part importante des formes organiques du phosphore particulaire. Les meilleures performances d'élimination du phosphore sont notées au printemps ( $77 \%$ pour les $\mathrm{PO} 4$ et $64 \%$ pour le phosphore total). La période la moins performante est la période automne-hiver où les rendements moyens ne dépassent pas 38\%. Le phosphore éliminé est stocké dans les sédiments. En période estivale, des phénomènes de relargage du phosphore à partir des sédiments ont été mis en évidence, favorisés par une zonation verticale des eaux à cette période. Des essais aulaboratoire ont montré la grande aptitude des sédiments de la station de lagunage étudiée à relarguer le phosphore, avec intervention de l'activité biologique du sédiment. 


\title{
Variations saisonnières des formes de phosphore dans une station de traitement des eaux usées par lagunage, sous climat aride de Marrakech (Maroc)
}

\author{
Seasonal variations in the forms of phosphorus \\ in a wastewater stabilization pond under arid climatic \\ conditions at Marrakech (Morocco)
}

\author{
N. OUAZZANI ${ }^{1}$, L. BOUARAB ${ }^{1}$, B. PICOT ${ }^{3}$, H.B. LAZREK ${ }^{2}$, B. OUDRA ${ }^{1}$ et J. BONTOUX ${ }^{3}$
}

Reçu le 16 décembre 1996, accepté le 18 août 1997*.

\section{SUMMARY}

Assimilation, sedimentation and release are the principal mechanisms controlling the behavior of phosphorus in aquatic ecosystems. There are numerous investigations of the phosphorus cycle in lakes and rivers in relation to the eutrophication process. There are, however, few studies about phosphorus cycling in waste stabilization ponds and most of these have discussed phosphorus removal.

The aims of this paper were: 1) to study phosphorus dynamics in relation to the biomass compartment (zooplancton, phytoplancton and bacteria); and 2) to evaluate the phosphorus removal efficiency of the treatment plant under arid climatic conditions. Of particular interest was the remobilization of phosphorus from the pond sediment, both under laboratory conditions and in the field.

The experimental installation consisted of two ponds receiving domestic wastewater (mean flow $=120.4 \pm 5.8 \mathrm{~m}^{3} \cdot \mathrm{day}^{-1}$ and specific organic load $=56 \mathrm{~kg}$ $\mathrm{BOD} \cdot \mathrm{ha}^{-1} \cdot \mathrm{day}^{-1}$; pond area $=0.5 \mathrm{ha}$ and depth $=1.5 \mathrm{~m}$ ). Samples were taken twice monthly from the surface, B1 (S) and B2 (S), and from the $1.5 \mathrm{~m}$ layers, B1 (F) and B2 (F). Composite ( 24 h) samples were taken from the inflow and the outflow of the plant to determine the removal efficiencies. In each sample, soluble reactive phosphorus (PO4), total phosphorus (PT) and total soluble phosphorus (PTD) were determined. Particulate phosphorus (PTP) was calculated as (PT-PTD) and soluble organic phosphorus (POD) as (PTD-PO4).

Phosphorus release was measured in a plexiglas flask containing sediment (col* lected by corer from the pond bottom) overlaid with distilled water. Over 15

1. Laboratoire d'hydrobiologie (Analyse et Ecotoxicologie), faculté des Sciences de Marrakech-Semlalia, Maroc.

2. Laboratoire de chimie organique appliquée, faculté des Sciences de Marrakech-Semialia, Maroc.

3. Département Sciences de l'environnement et santé publique, UMR 5556, Faculté de Pharmacie, avenue Ch. Flahault, 34060 Montpellier, France.

* Les commentaires seront reçus jusqu'au 20 juillet 1998. 
days, daily measurements were made of $\mathrm{PO}_{4}$ and total phosphorus. Sediment dry weight $(\%)$, volatile matter, organic carbon $(\%)$ and total phosphorus were measured before and after each test. Phosphorus contents of the phytoplankion, zooplankton and bacteria were estimated using the methods of BOU. GIS (1974).

The results show that cyclical and seasonal variations of phosphorus content were closely correlated with variations in the biological components. Significant correlation coefficients were noted between chlorophyll- $a$ and particulate organic phosphorus. Organic phosphorus in algal cells was the more important form of particulate organic phosphorus.

Under the arid climate, the waste stabilization ponds were more efficient at phosphate removal during the spring-summer period $\left(77 \%\right.$ of $\mathrm{PO}_{4}$ and $64 \%$ of total phosphorus in spring). The first pond may play the major role in phosphate removal. Phosphorus is only eliminated from stabilization ponds though accumulation in the sediment. However, part of the phosphorus which accumulates in pond sediments remains potentially mobile. During the summer period, phosphorus release from the sediment, observed in situ, was favored by stratification of the overlying water. The same phenomenon was noted during the autumn, particularly the release of POD. Laboratory studies have shown that phosphorus release may amount to $55 \mathrm{mg} \mathrm{P} \mathrm{m}^{-2} \mathrm{~d}^{-1}$. Biological activity may play a significant role in this mobilization.

Key-words : phosphorus, wastewater treatment, stabilization pond, removal efficiency, release.

L'objectif de ce travail est d'étudier dans un premier temps la dynamique du phosphore dans un système de traitement d'eaux usées par lagunage facultatif, en relation avec les compartiments biologiques : phytoplancton, zooplancton et bactéries, dans un deuxième temps, d'évaluer, sous les conditions du climat aride, les performances épuratoires de ce système en ce qui concerne les formes du phosphore. Un intérêt particulier est porté au phénomène du relargage du phosphore par les sédiments.

Les résultats montrent qu'il y a une évolution saisonnière et cyclique du phosphore dans les bassins de lagunage, en étroite corrélation avec le phytoplancton et le zooplancton. Le phytoplancton représente la part importante des formes organiques du phosphore particulaire. Les meilleures performances d'élimination du phosphore sont notées au printemps $\left(77 \%\right.$ pour les $\mathrm{PO}_{4}$ et $64 \%$ pour le phosphore total). La période la moins performante est la période automne-hiver où les rendements moyens ne dépassent pas $38 \%$. Le phosphore éliminé est stocké dans les sédimerts. En période estivale, des phénomènes de relargage du phosphore à partir des sédiments ont été mis en évidence, favorisés par une zonation verticale des eaux à cette période. Des essais au laboratoire ont montré la grande aptitude des sédiments de la station de lagunage étudiée à relarguer le phosphore, avec intervention de l'activité biologique du sédiment.

Mots-clés : phosphore, traitement des eaux usées, lagunage, performance épuratoire, relargage. 


\section{1 - INTRODUCTION}

Le cycle du phosphore dans un écosystème aquatique est régi par plusieurs processus complexes, décrits par BOSTRÖM et al. (1988), qui peuvent être résumés en une assimilation, une sédimentation et une régénération (SOMIYA et al., 1984).

Le phosphore, sous sa forme soluble bioassimilable, est un élément nutritif essentiel pour les algues. Celles-ci assimilent généralement le phosphore sous forme d'orthophosphates. Toutefois certaines espèces sont capables d'utiliser des formes organiques dissoutes. Les algues peuvent même consommer le phosphore en excès, c'est la consommation de luxe (PETTERSON et al., 1985). L'assimilation cellulaire dépend de leur capacité d'emmagasinement (HENRY et al., 1984), de la température et de l'éclairement. Les bactéries, elles, assimilent et immobilisent le phosphore pour leur propre compte, sous forme d'acides nucléiques, de phospho-lipides, de phospho-protéines, etc (MARTIN, 1987). Les bactéries, comme les algues, possèdent une consommation de luxe, qui peut être importante en imposant une alternance de phases anaérobies et aérobies (FLO. RENTZ et al., 1984).

Le phosphore organique particulaire des sédiments se trouve sous forme de bactéries, algues et zooplancton, molécules détritiques animales et végétales en décomposition ou en association insoluble avec des acides humiques (BOSTRÖM et al., 1984). La précipitation chimique des phosphates se fait principalement avec le fer, le calcium ou le manganèse. Ces précipitations chimiques dépendent du pH (STUMM et MORGAN, 1981) L'adsorption des phosphates sur les matières en suspension et sur les sédiments se fait par des liaisons physiques de type Van Der WAALS ou chimiques par échanges de ligands $\mathrm{OH}^{-}$des complexes hydroxométalliques (FeOOH)n (LIJKLEMA et al., 1980; BOSTRŌM et al., 1988) et $(\mathrm{AlOOH}) \mathrm{n}$ (GUNATILAKA et al., 1982) Elle est influencée par la température (BOERS et VAN HESE, 1988) et par le pH (LIJKLEMA, 1977 ; FOX et al., 1989), par la nature des sédiments (HOLDREN et ARMSTRONG, 1980) et par sa granulométrie (LEROUX et al., 1987).

La régénération du phosphore à partir des sédiments se fait par mobilisation biologique ou physico-chimique. Les bactéries en anaérobiose et les microalgues à l'obscurité changent de métabolisme et relarguent le phosphore stocké dans leur cytoplasme, dans le milieu extérieur (ROQUES, 1980 ; FLORENTZ et al., 1984 ; SOMIYA et al., 1988). Les bactéries peuvent égatement solubiliser les formes minérales insolubles du phosphore, en produisant des acides organiques ou catalyser la minéralisation du phosphore organique par production de phosphatases (MARTIN, 1987). Les cellules algales mortes s'autolysent et libèrent les phosphates dans l'eau (GOLTERMANet al., 1975. Le zooplancton intervient en excrétant dans le milieu extérieur, une partie du phosphore ingéré (PREMAZZl et al., 1982).

La température, le potentiel rédox et le pH sont les facteurs les plus importants à affecter la mobilisation du phosphore minéral à partir des sédiments (MARCIA et al., 1980 ; BOERS et al., 1990). Les différentes associations Fe-P, Al-P, sont plus facilement mobilisables que les autres formes particulaires précipitées, comme l'apatite, qui piègent le phosphore.

Afin d'élucider sa participation au phénomène d'eutrophisation, le cycle du phosphore a été généralement étudié dans des écosystèmes d'eaux douces en 
particulier les lacs (GOLTERMAN et al., 1981 ; ISTAVANOVICS, 1988 ; AFDALI et al., 1990), ou les étangs côtiers (MOUTIN et al., 1992). Les travaux concernant le cycle du phosphore sur une station de traitement d'eaux usées par lagunage sont assez rares et portent essentiellement sur l'aspect performance épuratoire. Au Japon, SOMIYA et al., (1984) ont montré que l'élimination du phosphore peut atteindre jusqu'à $43 \%$ dans un traitement tertiaire par lagunage après une station de boues activées. En France, MARTIN (1987), a décrit un rendement moyen de $53 \%$ dans une vingtaine de stations d'épuration par lagunage, RACAULT et al., (1995) à partir de 160 stations, donnent un taux d'élimination moyen du phosphore de $57 \%$. PICOT et al., (1992) trouvent un rendement d'élimination du phosphore de $19,5 \%$ seulement dans le bassin de lagunage de Mèze, sous climat méditerranéen, au sud de la France.

La plupart des travaux qui ont abordé la relation entre les différentes formes de phosphore et les algues ou le zooplancton ont été réalisés dans les lacs (DILLON and RIGLER, 1974 ; LEVINE and SCHINDLER, 1980 ; WELCH et al., 1990). La dynamique du phosphore dans les bassins de stabilisation a été très peu abordée, en particulier l'importance des phénomènes de régénération de cet élément à partir des boues déposées. SOULEMANE (1985), sous climat tempéré en France, a mis en évidence le relargage du phosphore à partir des sédiments de bassins de stabilisation. EL HALOUANI (1992), dans un lagunage à haut rendement, alimenté par des eaux usées particulièrement riches en calcium (125 mg/l), estime que l'élimination du phosphore se fait essentiellement par précipitation de phosphates de calcium, sous le contrôle du pH. À Marrakech, sous climat aride, les premières études sur le lagunage ont été entreprises depuis 1985, afin de comprendre les mécanismes qui contrôlent le devenir du phosphore dans ce type de système de traitement (BOUARAB et al., 1989).

L'objectif de ce travail est :

1) d'étudier la dynamique du phosphore, en relation avec les autres compartiments biologiques du système, notamment le phytoplancton, le zooplancton et les bactéries;

2) d'évaluer les performances épuratoires concernant le phosphore dans un système d'épuration des eaux usées par lagunage, sous climat aride. Un intérêt particulier sera porté aux phénomènes de relargage du $P$ à partir des sédiments, in situ et par des essais au laboratoire.

\section{2 - MATÉRIELS ET MÉTHODES}

\subsection{Site de l'étude}

La station expérimentale de traitement d'eaux usées étudiée reçoit une partie des eaux usées de la ville de Marrakech (Maroc, $31^{\circ} \mathrm{N}, 8^{\circ} 02 \mathrm{~W}$, altitude : $471 \mathrm{~m}$ ). Elle est constituée de deux bassins facultatifs en série (fig. 1). Les deux bassins ont une forme semi-circulaire, munis d'une île au centre.

Cette forme hydrodynamique permet d'éviter les écoulements préférentiels. La surface du plan d'eau est de 0,5 ha et la profondeur de $1,5 \mathrm{~m}$. 


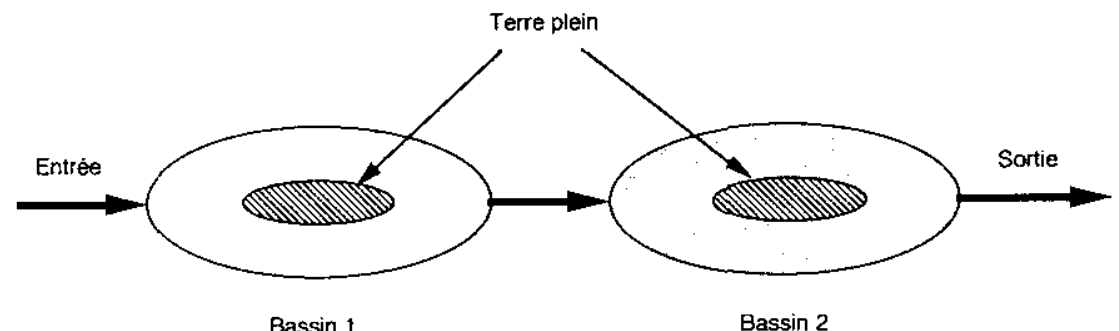

Figure 1 Schéma de la station de lagunage étudiée.

(Surface : 0,5 ha ; profondeur : $1,5 \mathrm{~m}$ ).

Schematic view of stabilization pond studied.

(Area: 0,5 ha; depth: $1,5 \mathrm{~m}$ ).

À l'entrée et à la sortie du système, des limnigraphes enregistreurs permettent une mesure continue du débit. La charge hydrique varie entre $(116,3$ $\left.\pm 8,7 \mathrm{~m}^{3} \cdot \mathrm{j}^{-1}\right)$ et $(124,5 \pm 5,7) \mathrm{m}^{3} \cdot \mathrm{j}^{-1}$ et égale en moyenne $120,4 \pm 5,8 \mathrm{~m}^{3} \cdot \mathrm{j}^{-1}$ (tabl. 1), ce qui correspond à 1000 équivalenthabitant, calculé sur la base d'un rejet moyen de 120 I par habitant et par jour (BENNIS, 1986). Compte tenu de l'évaporation mesurée qui varie de $3 \mathrm{~m}^{3} \cdot \mathrm{j}^{-1}$ en hiver à $46,8 \mathrm{~m}^{3} \cdot \mathrm{j}^{-1}$ en été, le temps de séjour a varié entre 45 et 60 jours.

Tableau 1 Bilan hydrique de la station durant les années 1986-1987.

Table $1 \quad H y d r a u l i c$ balance-sheet of the station during the years 1986-1987.

\begin{tabular}{|lcccccccccccccc|}
\hline & Année & J & F & M & A & M & J & J & A & S & O & N & D & Moy \pm er. std. \\
\hline Débit entré & 1986 & 130 & 124 & 124 & 122 & 131 & 136 & 128 & 116 & 117 & 124 & 121 & 119 & $124,5 \pm 5,7$ \\
$\left(\mathrm{~m}^{3} \cdot \mathrm{j}^{-1}\right)$ & 1987 & 111 & 128 & 111 & 108 & 123 & 123 & 126 & 126 & 118 & 102 & 107 & 113 & $116,3 \pm 8,7$ \\
Débit sortie & 1986 & 82 & 76 & 76 & 79 & 75 & 73 & 65 & 57 & 59 & 88 & 99 & 86 & $76,3 \pm 12$ \\
$\left(\mathrm{~m}^{3} \cdot \mathrm{l}^{-1}\right)$ & 1987 & 58 & - & 77 & - & 69 & 80 & 75 & 70 & 80 & 78 & 80 & 96 & $75,5 \pm 9,6$ \\
Évaporation $^{1}$ & 1986 & 18,2 & 21,6 & 24,3 & 26,6 & 39,9 & 46,8 & 46,8 & 43,0 & 42,0 & 20,0 & 6,0 & 17,0 & $32,0 \pm 14,9$ \\
$\left(\mathrm{~m}^{3} \cdot \mathrm{j}^{-1}\right)$ & 1987 & - & 3,0 & 12,0 & 22,4 & - & 40,0 & 40,0 & 20,0 & - & 14,8 & 13,0 & - & $20,7 \pm 13,3$ \\
Infiltration & 1986 & - & - & - & - & - & - & - & - & - & - & - & - & - \\
$\left(\mathrm{m}^{3} \cdot \mathrm{j}^{-1}\right)$ & 1987 & & - & 22,0 & 17,6 & - & 3,0 & 11,0 & 36,0 & - & 9,2 & 14,0 & - & $16,1 \pm 10,6$ \\
\hline
\end{tabular}

La charge organique superficielle reçue par la station est de l'ordre de $56 \mathrm{~kg}$. DBO5 ha-1. $\mathrm{j}^{-1}$.

L'échantillonnage est réalisé simultanément pour l'évaluation des paramètres physico-chimiques et biologiques à un rythme bimensuel durant deux ans. Les prélèvements d'au moins $1 \mathrm{I}$. réalisés dans les deux bassins, en surface $[B 1$ (S) et $\mathrm{B} 2(\mathrm{~S})]$ grâce à une perche et à 1 mètre de profondeur $[\mathrm{B} 1(\mathrm{~F})$ et $\mathrm{B} 2(\mathrm{~F})]$ grâce à une bouteille à clapet, sont conservés dans la glace jusqu'au laboratoire. Des prélèvements mensuels d'échantillons moyens de 24 heures à l'entrée et à la sortie de chaque bassin permettent de calculer les rendements épuratoires. 


\subsection{Paramètres mesurés et méthodes utilisées}

- Les paramètres physico-chimiques $\left(t^{\circ} \mathrm{C}, \mathrm{pH}\right.$, conductivité, oxygène dissous) sont mesurés sur le terrain grâce à une multisonde de type Horiba.

- Demande chimique en oxygène (DCO), Demande biologique en oxygène (DBO5), Matières en suspension (MES), Matières en suspension volatile (MVS), azote Kjeldahl sur échantillon brut (NTK) ont été dosés selon les méthodes de l'Association Française de Normalisation. La différence entre azote Kjeldahl effectué sur échantillon brut (NTK) et sur échantillon filtré (NTD) sur membrane Whatman GFC a permis de calculer l'azote total particulaire (NTP). Généralement, la distinction entre matériel dissous et particulaire se fait arbitrairement par filtration des échantillons sur membrane 0,45 micromètre. Ici la séparation a été effectuée par filtration sur filtre Whatman GFC, filtre préconisé pour déterminer les matières en suspension selon la norme AFNOR T 90 105. L'azote ammoniacal $\left(\mathrm{NH}_{4}\right)$ a été dosé par la méthode spectrophotométrique au bleu d'indophénol selon la norme T 90015.

- Les orthophosphates $\left(\mathrm{PO}_{4}\right)$ ont été mesurés selon la norme (T90-023), par formation en milieu acide $2<\mathrm{pH}<7$ d'un complexe phosphomolybdique avec le molybdate d'ammonium et le tartrate double d'antimoine et de potassium. Ce complexe est réduit par l'acide ascorbique en un complexe de coloration bleue, dont l'intensité est mesurée en spectrophotométrie à $700 \mathrm{~nm}$. Le dosage est effectué sur l'eau filtrée sur une membrane Whatman GF/C.

- Le phosphore total a été mesuré par minéralisation en milieu acide en présence de $\mathrm{K}_{2} \mathrm{~S}_{2} \mathrm{O}_{8}$, à $120^{\circ} \mathrm{C}$ à l'autoclave, sur des échantillons bruts (PT) et filtrés sur membranes Whatman GF/C (PTD). Le phosphore organique dissous (POD) est obtenu par différence (PTD- $\mathrm{PO}_{4}$ ) et le phosphore particulaire (PTP) est obtenu par différence (PT-PTD), ce phosphore particulaire peut être considéré dans les bassins de lagunage comme étant essentiellement du phosphore organique particulaire (POP).

Au laboratoire, l'étude du relargage du phosphore à partir des sédiments a été réalisée en mettant en contact des sédiments $(6 \mathrm{~cm}$ de hauteur), avec de l'eau distillée ( $8 \mathrm{~cm}$ de hauteur), dans des bacs de $36 \times 12 \times 20 \mathrm{~cm}^{3}$. Les sédiments ont été prélevés au fond du premier bassin du lagunage après 30 mois de fonctionnement de la station. Ce prélèvement est réalisé dans les premiers centimètres grâce à une carotte en PVC. Les teneurs des sédiments en matières volatiles, en carbone organique et en phosphore (PT) ont été évaluées au début et à la fin de l'expérience. Sur l'eau surnageante, les teneurs en PT et en $\mathrm{PO}_{4}$ ont été suivies pendant 15 jours.

Les données des paramètres biologiques sont extraites des travaux de OUDRA (1990) pour le phytoplancton et TIFNOUTI (1987) pour le zooplancton.

Le phosphore inclus dans la biomasse a été calculé en considérant $P=1,1 \%$ du poids sec pour le zooplancton, $P=0,5 \cdot 10^{-15} \mathrm{~g} /$ cellule pour les bactéries (TROUSSELIER, communication personnelle) et un gramme de chlorophylle correspond à une quantité de phosphore comprise entre 2,3 et $3,5 \mathrm{~g}$ (BOUGIS, 1974).

Une analyse en composantes principales a été réalisée sur 9 variables centrées réduites (Oxygène dissous, $\mathrm{pH}, \mathrm{MES}, \mathrm{PO}_{4}, \mathrm{PTP}, \mathrm{PTD}, \mathrm{NH}_{4}, \mathrm{NTP}, \mathrm{NTD}$ ) et sur 25 individus correspondant aux différents temps de prélèvements. Ces individus sont numérotés dans la figure 3 selon le mois de leur saisie. Une classification hiérarchique a été utilisée pour le regroupement des individus dans les différentes représentations selon les axes principaux. 


\section{3 - RÉSULTATS ET DISCUSSION}

\subsection{Dynamique du phosphore dans le lagunage}

Les caractéristiques physico-chimiques moyennes des eaux usées à l'entrée sont décrites au tableau 2. Le phosphore est composé de 50 à $70 \%$ de $\mathrm{PO}_{4}$, de 10 à $25 \%$ de POD et 20 à $25 \%$ de PTP.

Tableau 2 Caractéristiques moyennes des eaux usées brutes durant les années 19861987. Moyenne, écart-type de la moyenne, valeur minimale (Min) et maximale (Max), réalisés sur 52 échantillons.

Table 2 Means characteristics of raw waste water (during 1986-1987). Mean, standard deviation of the mean, minimum value and maximum value, for 52 samples.

\begin{tabular}{|lcccc|}
\hline & Min & Max & Moyenne & Écart-type de la moyenne \\
\hline Température & 12,4 & 33,4 & 23,94 & 0,77 \\
$\mathrm{pH}$ & 7,2 & 8,7 & 7,71 & 0,037 \\
$\mathrm{DCO}\left(\mathrm{mgO}_{2} /\right)$ & 77 & 945 & 348 & 23,9 \\
$\mathrm{DCO}$ filtré $\left(\mathrm{mgO}_{2} /\right)$ & 16 & 377 & 168 & 10,7 \\
$\mathrm{MES}(\mathrm{mg} /)$ & 30 & 480 & 167,5 & 11,3 \\
$\mathrm{MVS}(\mathrm{mgll})$ & 10 & 244 & 91 & 6,8 \\
$\mathrm{NH}_{4}(\mathrm{mgN} /)$ & 9,3 & 71,0 & 42,4 & 2,3 \\
$\mathrm{PO}_{4}$ (mgPl) & 3 & 13,5 & 7,34 & 0,32 \\
PTotal dissous $(\mathrm{mgP} /)_{\text {PTotal particulaire }}$ & 4,2 & 20 & 8,9 & 0,43 \\
\hline
\end{tabular}

Dans les deux bassins, les résultats obtenus sur deux années successives, 1986-87, montrent l'existence d'évolution cyclique annuelle pour les orthophosphates, que ce soit en surface ou à un mètre de profondeur (fig. 2). Une analyse en composantes principales (ACP), suivie d'une classification hiérarchique, a été réalisée sur les données physico-chimiques pour l'année 1987 (fig. 3). Pour le bassin 1 en surface, PTD, $\mathrm{PO}_{4}$ et MES contribuent majoritairement au premier axe (40\% d'inertie) tandis que NTP et $\mathrm{NH}_{4}$ définissent le second axe $(23 \%$ d'inertie). La position des individus dans l'espace formé par les deux premiers axes principaux permet de distinguer plusieurs périodes caractéristiques dans le fonctionnement de l'écosystème :

- une période de printemps (mars-avril-mai),

- une période d'été (juin-juillet-août),

- une période regroupant automne et hiver,

- deux mois de transitions s'isolent, septembre et février.

La figure 4 représente, pour chacune de ces périodes, les proportions relatives des orthophosphates $\left(\mathrm{PO}_{4}\right)$, du phosphore organique particulaire (POP) et dissous (POD), à l'entrée, dans les deux bassins, en surface B1 (S) et B2 (S) et en profondeur $B 1(F)$ et $B 2(F)$. Ces pourcentages sont ramenés par rapport aux teneurs en phosphore total à l'entrée du système de traitement, dans un but de rendre compte du devenir des différentes formes du phosphore. 

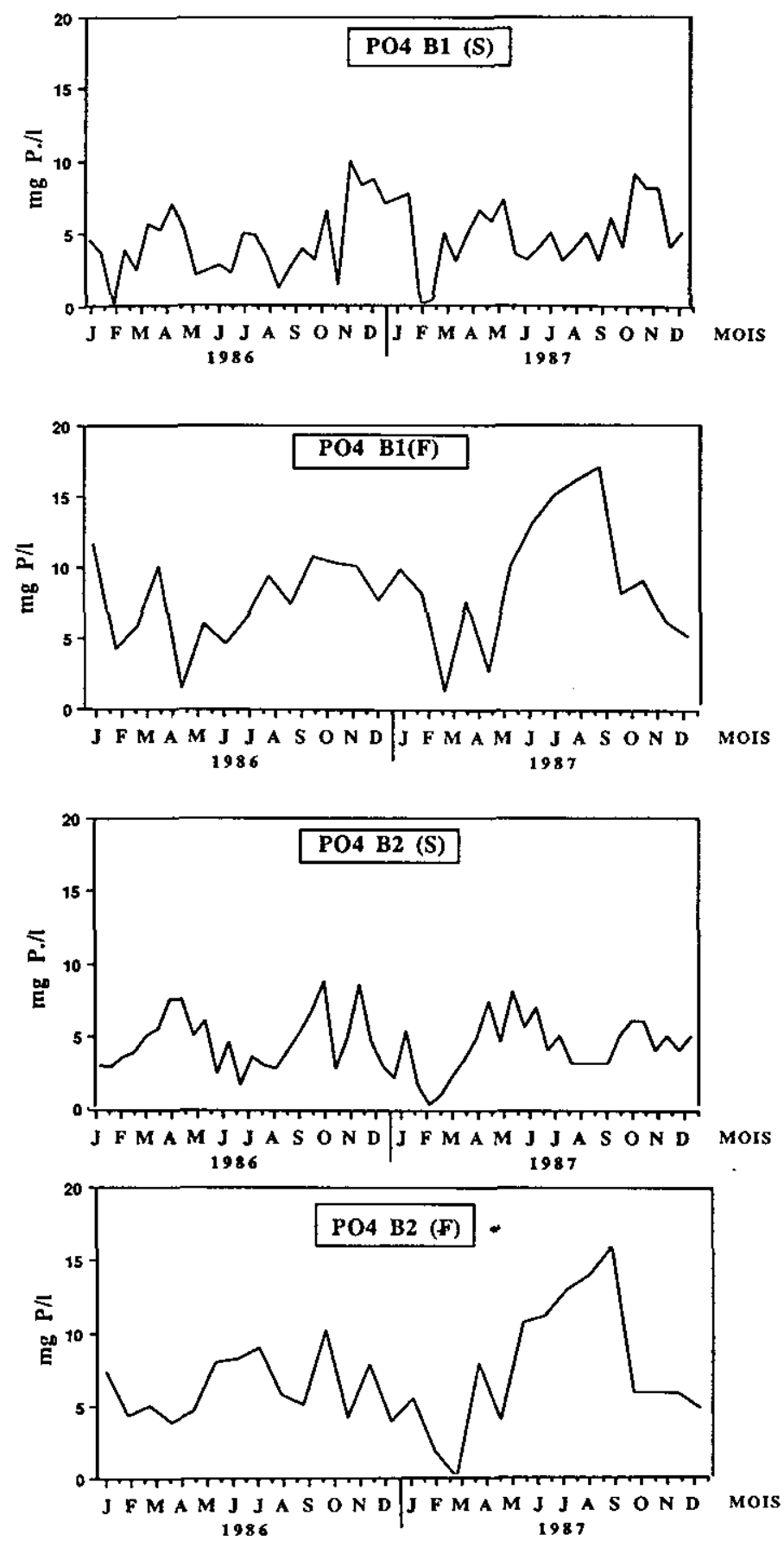

Figure 2 Évolution des orthophosphates dans les deux bassins en surface $B 1(S)$ et $B 2(S)$ et à 1 mètre de profondeur $B 1(F)$ et $B 2(F)$.

Evolution of dissolved reactive phosphate $\left(\mathrm{PO}_{4}\right)$ in both ponds, at the surface $[B 1$ (S) and $B 2$ (S)] and at $1 \mathrm{~m}$ depth [(B1 (F) and B2 (F)]. 


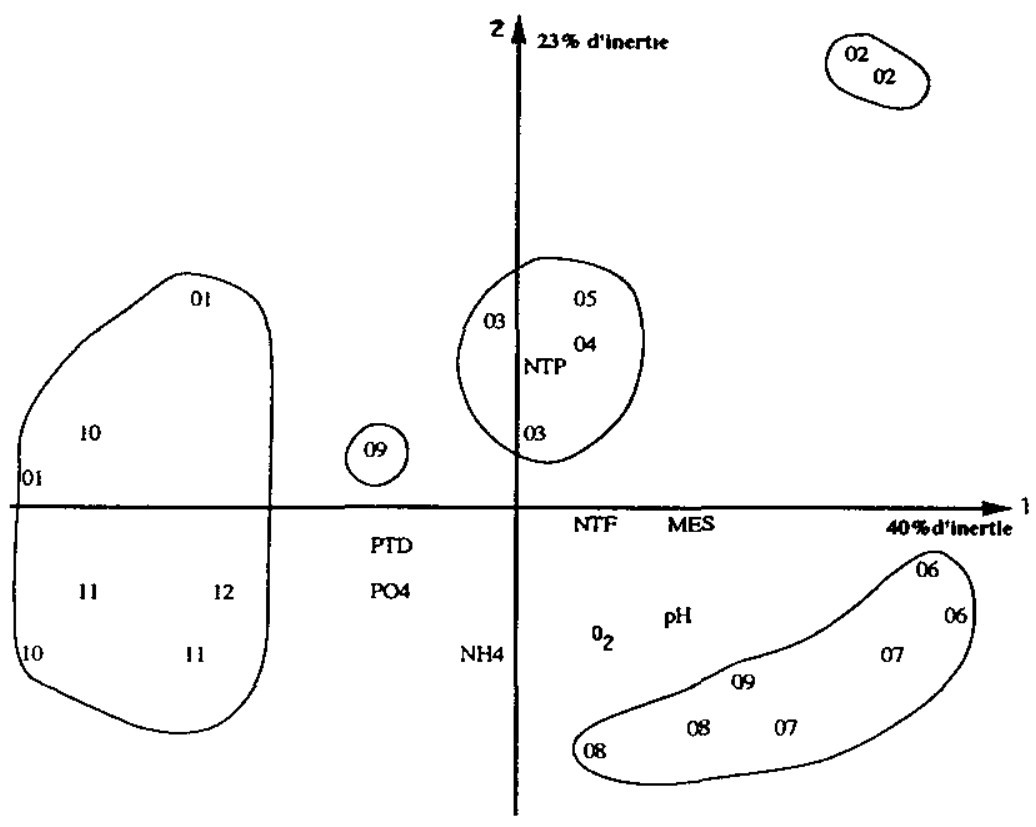

Figure 3 Représentation des 9 variables et des 25 individus dans le plan $1 \times 2(63 \%$ d'inertie) de l'analyse en composantes principales (Bassin 1 surface). Listes des variables et abréviations : $\mathrm{O}_{2}$ : oxygène dissous ; $\mathrm{pH} ; \mathrm{MES}$ : matières en suspension; $\mathrm{PO}_{4}$ : orthophosphates; PTP : phosphore total particulaire ; PTD : phosphore total dissous ; $\mathrm{NH}_{4}$ : azote ammoniacal; NTP : azote total particulaire ; NTD : azote total dissous. Les individus sont numérotés en fonction du mois de leur saisie : 01 pour janvier, 12 pour décembre.

Representation of 9 variables and 25 data points in the map of the first two components of the principal components analysis (PCA). List of variables and abbreviations: $\mathrm{O}_{2}$ : dissolved oxygen; $\mathrm{pH}$; MES: total suspended solids; PO4: dissolved reactive phosphorus; PTP: total particulate phosphorus; PTD: total dissolved phosphorus; NH4: ammonium nitrogen; NTP: total particulate nitrogen; NTD: total dissolved nitrogen.

\subsubsection{Période de printemps}

Elle peut être caractérisée par une prépondérance du POP dans les deux bassins, en surface comme en profondeur (fig. 4a). Les teneurs en chlorophylle a active sont élevées (1 $220 \mu \mathrm{g} / \mathrm{l}$ ) en surface du premier bassin (tabl. 3). D'ailleurs, les coefficients de corrélation entre la chlorophylle a et le POP sont égaux à $(r=0,556 ; n=14)$ pour le premier bassin et à $(r=0,547 ; n=14)$ pour le deuxième. $60 \%$ des orthophosphates présents à l'entrée ont été assimilés et transformés en phosphore organique particulaire. Les conditions de température et d'éclairement sont favorables au développement algal durant cette période mais également au développement des protozoaires.

Dans ces systèmes où se développent en symbiose, bactéries, algues et zooplancton, il est intéressant d'évaluer le phosphore inclus dans la biomasse de chacun de ces groupes. Les bactéries hétérotrophes aérobies, dont l'abondance 
Tableau 3 Teneurs moyennes en chlorophylle-a active et en phéopigments aux différentes périodes définies par l'ACP au cours de l'année 1987.

Table 3 Means values of active chlorophyll-a et pheophytins at different periods defined as by PCA for the 1987 field year.

\begin{tabular}{|cccccccccccc|}
\hline & \multicolumn{2}{c}{ Printemps (4) } & \multicolumn{2}{c|}{ Ėté (9) } & \multicolumn{2}{c|}{ Septembre (2) } & \multicolumn{2}{c|}{ Automne-hiver (7) } & \multicolumn{2}{c|}{ Fevrier (2) } \\
\hline & Chl aa & Pheo & Chl aa & Pheo & Chl aa & Pheo & Chl aa & Pheo & Chl aa & Pheo \\
\hline B1 (S) & 1219 & 910 & 1134 & 1170 & 320 & 1442 & 151 & 746 & 368 & 67 \\
& \pm 411 & \pm 478 & \pm 381 & \pm 307 & - & - & \pm 138 & \pm 288 & - & - \\
B1 (F) & 441 & 37 & 340 & 413 & 60 & 603 & 185 & 267 & 350 & 05 \\
& \pm 108 & \pm 14 & \pm 101 & \pm 150 & - & - & \pm 170 & \pm 162 & - & - \\
B2 (S) & 537 & 406 & 547 & 192 & 167 & 1234 & 477 & 1846 & 1251 & 85 \\
& \pm 142 & \pm 224 & \pm 105 & +71 & - & - & \pm 263 & \pm 600 & - & - \\
B2 (F) & 317 & 195 & 228 & 314 & 43 & 517 & 208 & 562 & 801 & 0 \\
& \pm 119 & \pm 128 & \pm 57 & \pm 109 & - & - & \pm 92 & \pm 201 & - & - \\
\hline
\end{tabular}

est de l'ordre de $10^{10}$ cellules/l (BOUSSAID, 1987) représentent moins de $1 \%$ de ce POP. Le zooplancton (104-106 individus/l) (TIFNOUTI, 1987 ; CANOVAS, 1991) représente au plus $5 \%$ du POP. Le phosphore contenu dans le phytoplancton représenterait de 20 à $30 \%$ du POP selon le facteur de conversion utilisé pour transformer la chlorophylle a en phosphore. En réalité, la part du phosphore d'origine algale est sous estimée dans ces calculs, en raison de la part importante de phéopigments présents dont il n'a pas été tenu compte.

\subsubsection{Période d'été : (fig. 4b)}

On assiste à une stratification verticale dans les bassins, pour toutes les formes du phosphore. Cette stratification se retrouve pour la température, le $\mathrm{pH}$ et l'oxygène dissous (BOUARAB, 1988).

En surface, le POP est majoritaire dans le premier bassin. La teneur élevée en chlorophylle a confirme l'origine algale de ce phosphore. Le coefficient de corrélation calculé entre les teneurs du POP et ceux de la chlorophylle a est significatif $(r=0,753 ; n=9)$ dans le premier bassin et hautement significatif $(r=0,834$; $n=9$ ) dans le cas du deuxième bassin.

Bien que les rotifères présentent des densités nettement plus importantes qu'au printemps, la part de phosphore contenue dans le zooplancton reste inférieure à $5 \%$ du phosphore particulaire.

En profondeur, le pourcentage élevé des $\mathrm{PO}_{4}$, qui dépasse même le phosphore total à l'entrée, traduirait d'une part une minéralisation accrue des cellules algales mortes et, d'autre part, un relargage des $\mathrm{PO}_{4}$ à partir des sédiments.

L'élévation des teneurs en phéopigments (tabl. 3) et les conditions d'anaérobiose qui règnent à partir de $50 \mathrm{~cm}$, confirment l'existence d'une dégradation des cellules algales. Selon GOLTERMAN, (1975), dans un lac, ces phénomènes peuvent avoir lieu aussi bien à l'épilimnion qu'à l'hypolimnion. La vitesse de sédimentation des cellules algales détermine la durée de leur décomposition dans la colonne d'eau. Selon REYNOLDS, (1984), plusieurs facteurs déclenchent la morta- 
lité des cellules algales: l'absence de lumière, support de la photosynthèse, la déficience en nutriments, une exposition aux substances toxiques ou enfin une infection par les champignons, les bactéries et les virus. La plupart de ces facteurs se trouvent réunis dans un bassin de lagunage.

D'autre part, le $\mathrm{pH}$ acide $(6,2)$, le potentiel rédox réducteur des boues $\left(\mathrm{EH}_{\mathrm{H}}\right.$ $=-100 \mathrm{mV}$ ) suite à une stratification des eaux ainsi que les températures élevées à ces mois de l'année, sont favorables au processus de relargage des phosphates à partir des sédiments (GOLTERMAN et al., 1982). Le même relargage estival a été observé par SOULEMANE, (1985) dans le lagunage de la Chapelle Thouarault, en France.

Une étude, réalisée au laboratoire, a montré que les sédiments prélevés dans cette même station de lagunage ont une grande aptitude à relarguer le phosphore. Les flux journaliers du phosphore émanant des sédiments vers l'eau surnageante sont de l'ordre de $55 \mathrm{mg} \mathrm{P} \cdot \mathrm{m}^{-2} \cdot \mathrm{j}^{-1}$; ISTAVANOVICS (1988) a trouvé des flux de $\mathrm{PO}_{4}$ pouvant atteindre $4 \mathrm{mg} \mathrm{P} \cdot \mathrm{m}^{-2} \cdot \mathrm{j}^{-1}$ dans des zones hypertrophiques d'un lac de faible profondeur.

Le tableau 4 présente la teneur en matière organique, exprimée en pourcentage de matières volatiles et en \% de carbone par rapport au sédiment sec et la teneur en phosphore des sédiments, en début et à la fin de l'expérimentation. La matière organique et le phosphore total du sédiment diminuent au cours du temps, ce qui montre une minéralisation de la matière organique. La mobilisation du phosphore peut s'expliquer par la minéralisation en milieu oxique et par la désorption des oxydes de fer en milieu réducteur. Les oxohydroxydes de fer, sur lesquels sont adsorbés les orthophosphates, sont utilisés, en absence d'oxygène et de nitrate, comme accepteurs d'électrons dans le processus de minéralisation, entraînant la libération des $\mathrm{PO}_{4}$ (MOUTIN, 1992). Par ailleurs, les acides faibles, produits par la dégradation anaérobie, semblent également stimuler le relargage du phosphore (TAM et al., 1992).

Tableau 4 Teneurs en matières organiques (\%), en carbone organique (\%), en humidité $(\%)$ et en phosphore total du sédiment de lagunage avant et après test de relargage.

Table 4 Organic matter, organic carbon, dry weight and total phosphorus content of pond bottom sediment, determined before and after the release tests.

\begin{tabular}{|lcccc|}
\hline & \multirow{3}{*}{ Avant relargage } & \multicolumn{3}{c|}{ Après tests de relargage } \\
\cline { 3 - 5 } & & $\mathbf{2} \mathrm{cm}$ & $\mathbf{4} \mathrm{cm}$ & $\mathbf{6 c m}$ \\
\hline$\%$ Eau & $47 \%$ & $62 \%$ & $54 \%$ & $44 \%$ \\
$\%$ Matières volatiles & $69 \%$ & $50 \%$ & $58 \%$ & $56 \%$ \\
$\%$ Carbone organique & $38 \%$ & $18 \%$ & $27 \%$ & $30 \%$ \\
$\begin{array}{l}\text { Phosphore total } \\
\mathrm{mg} \mathrm{P} \cdot \mathrm{g}^{-1} \text { sédiment sec }\end{array}$ & 20 & 17 & 18 & 19 \\
$\mathrm{PO}_{4}$ de l'eau interstitielle & 15 & 12 & 13,6 & 14,2 \\
$\mathrm{mgP} \cdot \mathrm{I}^{-1}$ & & & & \\
\hline
\end{tabular}


P/PT Entrée

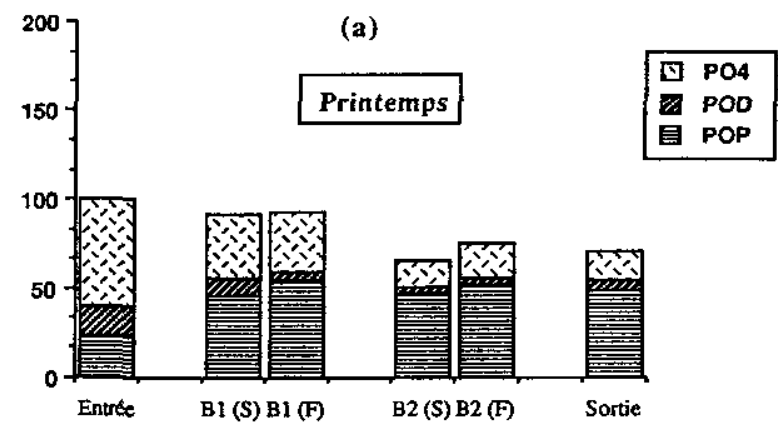

\% P/PT Entrée

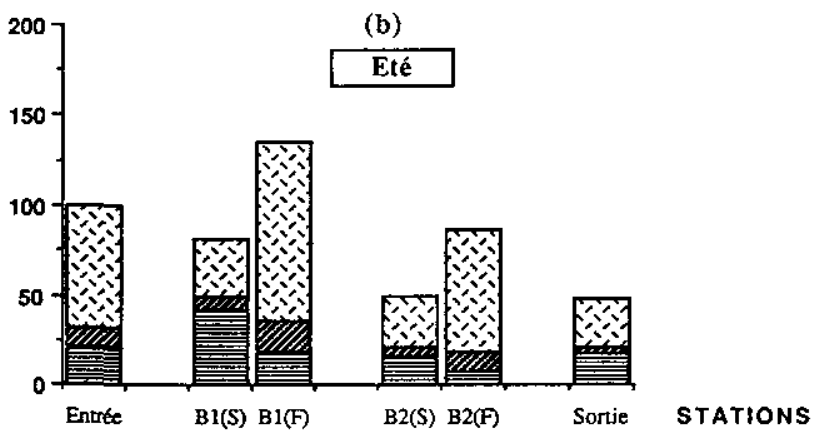

\% P/PT Entrée

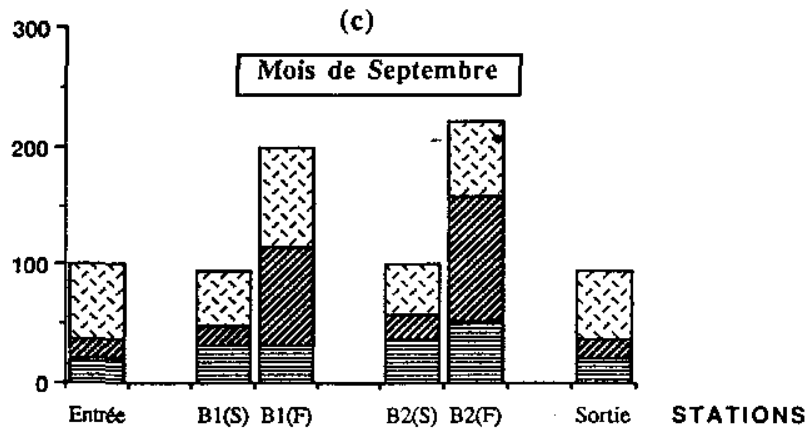




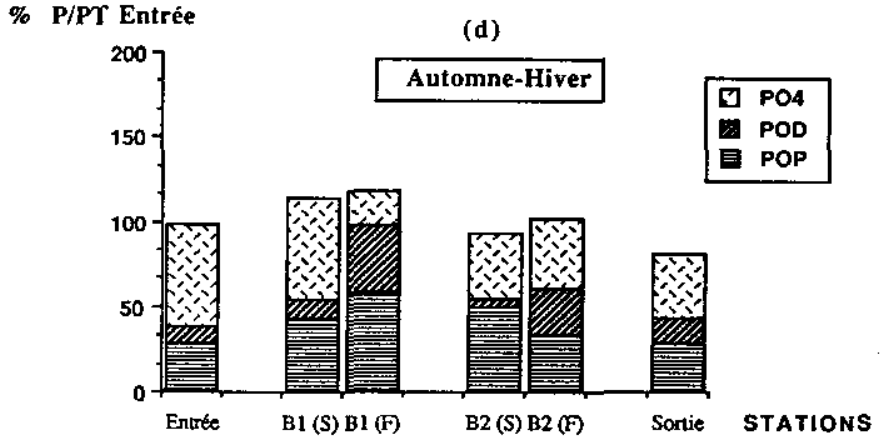

\% P/PT Entrée

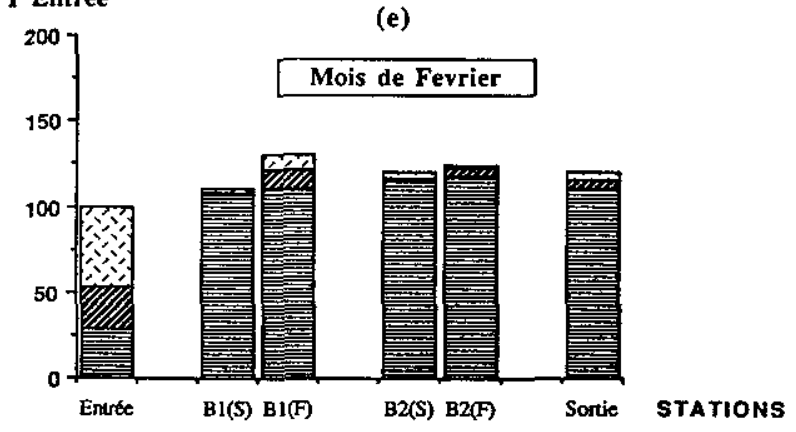

Figure 4 Proportions relatives établies par rapport au phosphore total à l'entrée (P/Pt entrée) pour les orthophosphates (PO4), le phosphore organique particulaire (POP) et le phosphore organique dissous (POD). Les proportions sont montrées pour les périodes de l'année telles que définies par l'ACP : (a) printemps, (b) été, (c) septembre, (d) autome-hiver, (e) février. Convention : Bassin 1 et 2 en surface, $B 1(S)$ et $B 2(S)$; à $1 \mathrm{~m}$. de profondeur, $B t(F)$ et $\mathrm{B} 2(\mathrm{~F})$.

Proportions of dissolved reactive phosphorus (PO4), particulate organic phosphorus (POP) and dissolved organic phosphorus as \% of total phosphorus in the raw waste water, at each period as defined by PCA: (a) spring; (b) summer; (c) September; (d) autumn-winter; (e) February. Convention: Surface samples from ponds 1 and 2, B1(S) and B2(S); samples from $1 \mathrm{~m}$ depth from ponds 1 and $2, B 1(F)$ and $B 2(F)$.

\subsubsection{Mois de septembre: (fig. 4c)}

II se caractérise par des teneurs en POD très élevées en profondeur des deux bassins qui sont encore stratifiés. Au fond, les teneurs en phosphore sont nettement plus élevées qu'en surface et restent supérieures au phosphore total à l'entrée. Il semblerait que le relargage du phosphore en provenance des sédiments soit essentiellement sous forme de phosphore organique dissous. La diminution de l'intensité du rayonnement solaire, défavorable au développement algal, entraîne une augmentation nette des phéopigments au dépend de la chlorophylle a active (tabl. 3). L'autolyse des cellules algales expliquerait l'enrichissement en 
POD au fond des bassins. Contrairement à la période d'été, suite à la chute des températures affectant les processus bactériens, la minéralisation du POP semble être incomplète et n'aboutit pas jusqu'au $\mathrm{PO}_{4}$.

\subsubsection{Période Automne-hiver}

On assiste à une homogénéisation du phosphore dans les bassins (fig. $4 d$ ). Cette déstratification se retrouve également pour la température, le $\mathrm{pH}$ et l'oxygène dissous (BOUARAB, 1988).

Cette période correspond au développement des bactéries du cycle de soufre, qui communiquent à l'eau une couleur rouge. Ces " eaux rouges " apparaissent annuellement en novembre et décembre et pourraient concourir à limiter les populations algales. D'ailleurs, CHIFAA (1987), a pu constater une diminution de la diversité du phytoplancton dans ce même lagunage où seuł le genre Chlamydomonas subsiste en cette période de l'année.

Les faibles valeurs de température et d'intensité du rayonnement solaire limitent également l'activité algale. En effet, le tableau 111 montre les plus faibles teneurs en chlorophylle a active par rapport aux autres périodes de l'année. Aucune corrélation significative n'a été obtenue entre les différentes formes du phosphore et la chlorophylle a, de même qu'entre phosphore et phéopigment. Les formes du phosphore subissent des transformations indépendantes du phytoplancton dans les bassins.

\subsubsection{Mois de février}

Après les conditions défavorables en automne-hiver, l'augmentation de l'éclairement entraîne une reprise de l'activité algale qui se traduit par une forte assimilation des $\mathrm{PO}_{4}$. Pratiquement tout le phosphore se trouve sous forme de phosphore organique particulaire (fig. $4 e$ ). En effet, le nombre de cellules algales passe de $10^{7}$ à $10^{9}$ cellules par litre pour les deux bassins (OUDRA, 1990).

\subsection{Performances épuratoires}

En raison de l'importance du temps de séjour (45 à 60 j.) et de l'intensité de l'évaporation, qui, en été, peut atteindre $37 \%$ du volume d'eaux usées entrant dans la station, les calculs de rendements ont été réalisés en considérant les charges de phosphore à l'entrée et à la sortie des bassins. Le tableau 5 donne les rendements moyens sur deux années, et par saison, pour le premier bassin et l'ensemble de la station. Au Printemps, en raison de l'importance de lactivité algale, les rendements sont de $77 \%$ en $\mathrm{PO}_{4}$ et de $64 \%$ en phosphore total. En été, en raison de la régénération du phosphore à partir des sédiments et après photolyse des cellules algales, ces rendements ont légèrement diminué $(72 \%$ en $\mathrm{PO}_{4}$ et $56 \%$ en PT). La majorité de l'élimination du phosphore durant le printemps et l'été se fait dans le premier bassin. En fait, la croissance algale est si forte dans ce bassin que la population algale s'autosature en nourriture et en lumière, et la mortalité augmente. En automne, la diminution de l'activité algale et une remontée des boues entraînent une baisse des rendements en orthophosphates $(45 \%)$ et en phosphore total $(37 \%)$. Cette baisse des rendements est due principalement au mauvais fonctionnement du premier bassin. Les rendements du deuxième bassin à cette période ne semblent pas différents de ceux obtenus en été ou même ceux du printemps où ils ne dépassent pas $30 \%$. 
Tableau 5 Rendements épuratoires moyens par saison durant les années 1986-1987 pour les orthophosphates (PO4), phosphore total dissous (PTD) et phosphore total (PT) pour le premier bassin (B1) et pour l'ensemble des deux bassins $(B 1+B 2) . n=$ nombre de prélèvements moyens sur 24 heures utilisé pour le calcul des rendements saisonniers.

Table 5 Mean percent removal efficiencies for each season during the years 19861987 for dissolved reactive phosphorus (PO4), total dissolved phosphorus (PTD) and total phosphons (PT), for the first pond (B1) and for the two ponds together $(B 1+B 2) . n=$ number of 24 hour average samples used to calculate the seasonal removal efficiencies.

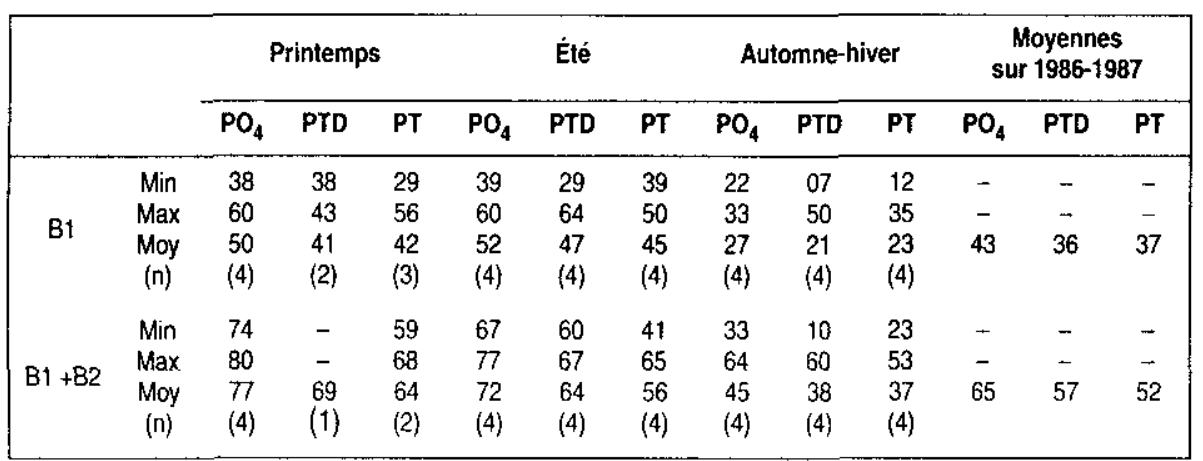

Enfin, les rendements annuels moyens $\left(65 \%\right.$ pour $\mathrm{PO}_{4}, 57 \%$ pour PTD et $52 \%$ pour PT) sont voisins de ceux cités par MARTIN (1987), sur une vingtaine de stations de lagunage françaises.

\section{CONCLUSION}

Ce travail présente les résultats de deux années d'investigation sur la station d'épuration des eaux usées par lagunage facultatif, sous climat aride de Marrakech.

Le suivi des paramètres phosphorés a permis dē défecter une évolution cyclique et saisonnière caractérisée par une dynamique du phosphore, en étroite relation avec les compartiments biologiques du système. Le phytoplancton représente la part la plus importante du phosphore organique particulaire.

En période printanière, le démarrage se fait d'une manière remarquable au mois de février où $100 \%$ du phosphore se trouve sous forme organique particulaire témoignant d'une forte assimilation algale. Le même phénomène se poursuit aux autres mois de la saison, avec seulement $60 \%$ du $\mathrm{PO}_{4}$ qui se transforme en POP suite au stockage de phosphore à l'intérieur des cellules. Le POP présente des corrélations significatives avec les valeurs de la chlorophylle a dans les deux bassins.

En période estivale, nous avons pu mettre en évidence une stratification verticale dans les bassins. En surface, la forte corrélation entre le POP et la chloro- 
phylle a est conservée. En profondeur, les fortes teneurs de $\mathrm{PO}_{4}$ témoignent d'une mortalité des cellules algales ou/et d'un relargage de $\mathrm{PO}_{4}$ à partir des sédiments. D'ailleurs, en laboratoire, les flux de phosphore à partir des sédiments du lagunage de Marrakech ont été évalués à $55 \mathrm{mg} \mathrm{P} \mathrm{m}^{2} \cdot \mathrm{j}^{-1}$.

La période automne-hiver a eu lieu juste après septembre, mois de transition où les fortes teneurs du phosphore organique dissous et des phéopigments témoignent d'une autolyse des cellules algales, qui se prolonge pendant le reste de la saison, suite aux conditions défavorables, surtout dans le premier bassin.

Ainsì, le lagunage, sous climat aride de Marrakech, présente des périodes de bon et de mauvais fonctionnement concernant l'élimination du phosphore. II présente les meilleures performances épuratoires en saisons printanières, avec des rendements allant jusqu'à $77 \%$ pour les $\mathrm{PO}_{4}$ et $64 \%$ pour le PT. La période la moins performante est la période d'automne-hiver où les rendements ne dépassent guère $45 \%$ pour les $\mathrm{PO}_{4}$. Le rôle du premier bassin s'est révélé primordial dans l'épuration. Le deuxième bassin n'assure qu'un second rôle avec des rendements assez constants durant toute l'année, ne dépassant guère les $30 \%$.

\section{REMERCIEMENTS}

Ce travail a été financièrement supporté par la coopération Franco-marocaine (Actions intégrées Franco-marocaine $n^{\circ} 75 / 83$ et $n^{\circ} 401 / 89$ ).

\section{RÉFÉRENCES BIBLIOGRAPHIQUES}

AFDALI, M., OUAZZANI, N., LOUDIKI, M. (1991). Spéciation et biodisponibilité du phosphore particulaire dans le lac réservoir Hassan $1^{\text {er }}$ et ses affluents (Maroc) "Hommage à F.A. Forel ": actes de la troisième conférence internationale d'expression française. Edit. J. P. Vernet, Morges (Suisse) : 163-166.

AFNOR (1994). Recueil des Normes Françaises: Qualité de l'eau, Environnement. Paris.

BENNIS-BENKIRANE, S (1986). Aspects physiologiques de la dégradation des hydrocarbures au laboratoire et dans le milieu naturel par des microflores de sol, d'eaux douces et d'eaux usées. Thèse de doctorat ès sciences, Paris VIt.

BOERS, P.C.M., VANHESE, O. (1988). Phosphorus release from the peaty sediments of the Loosdrecht lakes (The Netherlands), Wat. Res. 22 : 355-363.

BOERS, P.C.M. (1991). The influence of $\mathrm{pH}$ on ghosphate release from lake sediments, Wat. Res. $25: 309-311$.

BOSTRÖM, B. (1984). Potentiel mobility of phosphorus in different types of lake sediment. Int. Rev. Ges. Hydrobiol. 69 : 457 474.

BOSTRÖM, B., ANDERSEN, J.M., FLEISCHER, S., JANSSON, M. (1988). Exchanges of phosphorus across the sediment water interface. Hydrobiologia, $170: 229$. 244.

BOUARAB, L. (1988). Contribution à l'étude des différentes formes du phosphore dans le lagunage naturel : station expérimentale de Marrakech. Thèse de $3^{e}$ cycle. 
Université Cadi Ayyad. Marrakech, Maroc. $125 \mathrm{p}$.

BOUARAB, L., OUAZZANI, N., LAZREK, H.B., BONTOUX, J. (1989). Évolution des différentes formes du phosphore dans une station d'épuration par lagunage. $2^{e}$ congrés national de chimie. Marrakech 19-21 janvier.

BOUGIS, P. (1974). Écologie du plancton marin. Tome II: Le zooplancton. Masson \& Cie éd. Paris. $200 \mathrm{p}$.

BOUSSAID, A. (1987). Évolution des bactéries pathogènes: Salmonella et aéromonas en comparaison avec les bactéries témoins de contamination fécale dans des bassins expérimentaux d'épuration par lagunage sous climat aride. Thèse de $3^{e}$ cycle. Université Cadi Ayyad. Marrakech, Maroc.

CANOVAS, S. (1991). Structure du zooplancton en traitement des eaux usées par lagunage à haut rendement. Thèse de doctorat, Université Montpellier I. $170 \mathrm{p}$.

CHIFAA, A. (1987). Étude de la dynamique des peuplements phytoplanctoniques et interactions avec la qualité de l'eau en bassins expérimentaux de lagunage sous climat aride. Marrakech. Thèse de $3^{e}$ cycle. Université Cadi Ayyad, Marrakech Maroc.

DILLON, P.J., RIGLER, F.H. (1974). The phosphorus chlorophyll relationship in lakes. Limnology and Oceanography: $19: 767$. 773.

EL HALOUANI H. (1990). Lagunage à haut rendement: caractérisation physico-chimique de l'écosystème, étude de son aptitude à l'élimination de l'azote et du phos. phore dans l'épuration des eaux usées. Thèse de doctorat, Université Montpellier I. $154 \mathrm{p}$.

FLORENTZ, M., HARTEMANN, P., GILLES, P. (1984). Expérimentation sur pilote à alternance de phases portant sur f'élimination biologique du phosphore. Rev. Franç. des Sci. de l'eau, $3:$ 1-17.

FOX, I., MALATI, M. A., PERRY, R. (1989). The adsorption and release of phosphate from sediments of river receiving sewage effluent. Wat. Res., vol.23 : 725-732.

GOLTERMAN, H.L. (1975). Physiological limnology. An approch to physiology of lake ecosystems. Elsevier Scientific Publishing company, $488 \mathrm{p}$.
GOLTERMAN, H. L., SLYP, G., THOMAS, R.L. (1981). Study of the relationship between water quality and sediment transport. UNESCO, $231 \mathrm{p}$.

GUNATILAKA, A. (1982). Phosphate adsorption kinetics of resuspended sediments in shallow lake Heusieldlersee, Austria. Hydrobiologia 91 : 293-298.

HENRY, R., TUNDISI, J.G., CURI, P.R. (1984). Effects of phosphorus and nitrogen enrichment on the phytoplankton in tropical reservoir (Lobo reservoir, Brazil) Hydrobiologia 118 : 177-185.

HOLDREN, G.C., ARMSTRONG, D.E. (1980). Factors affecting phosphorus release from intakt lake sediment cores. Envir. Sci. Technol., $14: 79-87$.

ISTAVANOVICS, V. (1988). Seasonal variation of phosphorus release from the sediments of shallow lake Balaton (Hungary). Wat. Res. 22 : 1473-1481.

JENKINS, D., TANDOI, V. (1991). The applied microbiology of enhanced biological phosphate removal. Wat. Res., 25 : 1471-1478.

LEROUX, E., FEHRI, A., DORIOZ, J.M., BLANC, P. (1987). Contribution à l'étude des échanges de phosphore entre l'eau et les sédiments dans un système fluviolacustre. Sciences de l'eau, $6: 97-106$.

LEVINE, S.N., SCHINDLER, D.W. (1980). Radiochemical analusis of orthophos. phate concentration and seasonal changes in the flux of orthophosphate to seaston in two canadien Shield lakes. Can. J. Fish. Aquat. Sci. 37 : 479-489.

LIJKLEMA, L. (1980). Interaction of orthophosphate with iron (III) and aluminium hydroxydes. Environ. Sci. Technol., 14 : 537-54L.

LIJKLEMA, L. (1977). The role of iron in the exchange of phosphate between water and sediment. In: Golterman H.L. (ed.). Interactions between sediments and freshwater. Dr $W$. Junk Publishers. The Hague, 313-317

MARCIA, H., NEAFUS, J.E. (1980). Phosphorus release from sediments from lake Carl Blackwell, Oklahoma. Wat. Res., 14: 1477-1481.

MARTIN, G. (1987). Point sur l'épuration et et le traitement des effluents (eau, air), phosphore. vol. 3. Édit. Technique et Documentation. Lavoisier, Paris. 
MESNAGE, V. (1994). Contribution à l'étude de la mobilité des formes de phosphate à l'interface eau-sédiment dans des écosystèmes lagunaires. Thèse de doctorat, Université Montpellier I, $252 \mathrm{p}$.

MOUTIN, T., GAL, J.Y., EL HALOUANI, H., PICOT, B., BONTOUX, J. (1992). Decrease of phosphate concentration in high rate pond by precipitation of calcium phosphate: theoretical and experimental results. Wat. Res., 26 : $1445-1450$

MOUTIN, T. (1992). Contribution à l'étude du cycle du phosphate dans les écosystèmes lagunaires. Thèse de doctorat, Université Montpellier II. 250 p.

OUDRA, B. (1990). Bassin de stabilisation anaérobie et aérobie facultatif pour le traitement des eaux usées de Marrakech. Dynamique du phytoplancton (microplancton et picoplancton) et évaluation de la biomasse primaire. Thèse de $3^{e}$ cycle. Université Cadi Ayyad, Marrakech, Maroc, $144 \mathrm{p}$.

PETERSON, K. (1985). The availability of phosphorus and species composition of the spring phytoplankton in lake Erken. Int. Revue Ges. Hydrobiol. 70 (4) : 527546.

PICOT, B., BAHLAOUI, A., MOERSIDIK, S., BALEUX, B., BONTOUX, J. (1992). Comparison of purifing efficiency of high rate algal pond with stabilization pond. Wat. Sci. Tech., $25:$ 197-206.

PREMAZZl, G., MARENGO, G. (1982). Sedimentation rates in Swisse-Italian lake measured with sediment traps. Hydrobiologia, 92 : 603-610.

RACAULT, Y., BOUTIN, C., SEGUIN, A. (1995), Waste stabilization ponds in France: a report on fifteen years experience. Wat. Sci. Tech.. 31, 12 : 91-101
REYNOLDS, C.S. (1984). The ecology of freshwater phytoplancton. Cambridge University press, London, $384 \mathrm{p}$.

ROQUES, M. (1980). Fondements théoriques de traitement biologique des eaux. vol. II ed. Technique et Documentation. Paris

SOMIYA, I., SHIGEOFUJI, I. (1984). Materiel balances of organics and nutrients in an oxidation pond. Wat. Res. $18: 325-333$.

SOMIYA, I., TSUNO, H., MATSUMOTO, M. (1988). Phosphorus release-storage reaction and ogranic substrate behavior in biological phosphorus removal. Wat. Res. 22 : 49-58.

STUMM, W., MORGAN, J. (1981). Aquatic chemistry. An introduction emphasizing chemical equilibria in natural waters. $2^{\text {nd }}$ ed. Wiley Interscience Publication. NewYork, $780 \mathrm{p}$.

SOULEMANE, B. (1985). Évolution de nutriments dans une station de lagunage. Thèse $3^{e}$ cycle, Université de Rennes I, UER, ENSC de Rennes, $195 \mathrm{p}$.

TAM, N.F.Y., WONG, U.S., LEUNG, G. (1992). Effect of exogenous carbon sources on removal of inorganic nutrient by the nitrification-denitrification process. Wat. Res. $26: 1229-1236$

TIFNOUTI, A. (1987). Zooplancton de bassins de lagunage de Marrakech. Structure du peuplement et dynamique des principales populations. Thèse de $3^{e}$ cycle, Université Cadi Ayyad, Marrakech, Maroc.

WELCH, H.E., LEGAULT, J.A., KLING, H.J. (1989). Phytoplancton nutrients and primary production in fertilized and natural lakes at Saqvaqjuac, N.W.T. Can. J. Fish. Aquat. Sci. $46: 90-107$. 
COURTE NOTE

\title{
Évolution récente du régime hydrologique de quelques rivières de Roumanie centrale
}

SHORT NOTE

\author{
Recent hydrological trends \\ of some central Romanian rivers
}

J.P. CARBONNEL ${ }^{1}$, R. PETRORIAN² ${ }^{2}$ P. P. SERBAN²

Reçu le 3 décembre 1996, accepté le 12 juin 1997*.

SUMMARY

A statistical study of annual and seasonal discharges series for 15 Romanian rivers and the Danube River at Orsova exhibits a very important dry hydrological period which began around 1983. The winter discharge decreased $50 \%$ during this period and seems to be the main cause of this hydrological drought. The spatial distribution of this drought is defined in Central Romania. The analysis of the Danube discharge serie demonstrates that an important part of its watershed is in the same situation.

Key words: climatic evolution, hydrological drought, ammal discharges, stationarify, south Europe.

RÉSUMÉ

L'étude de la stationnarité de séries de débits annuels et saisonniers de 15 rivières roumaines et du Danube à Orsova met en évidence l'existence d'une « phase hydrologique » sèche importante depuis 1983. L'écoulement hivernal a diminué de $50 \%$ depuis cette date et semble principalement responsable de cette sécheresse hydrologique. L'extension géographique de cette sécheresse est précisée en Roumanie Centrale. L'analyse des débits du Danube montre qu'une partie importante de son bassin versant est touchée par le phénomène.

Mots-clés : évolution climatique, sécheresse hydrologique, débits annuels, stationnarité. Europe du sud.

1. Université P. et M. Curie, URA-CNRS 1367 , Case 123,4 place Jussieu, F-75252 PARIS Cedex 05.

2. Régie Autonome "Les Eaux Roumaines ", Dpt. Ressources en Eau, Str. Edgar Quinet 6, Bucuresti 70106, Roumanie.

* Les commentaires seront reçus jusqu’au 20 juillet 1998. 